\title{
Glucocorticoid Hyper- and Hypofunction:
}

\author{
Stress Effects on Cognition and Aggression
}

JEANSOK J. KIMa and JÓZSEF HALLER ${ }^{b}$

a Department of Psychology and Program in Neurobiology and Behavior, University of Washington, Seattle, Washington, USA

b Institute of Experimental Medicine, Hungarian Academy of Science, Budapest, Hungary

\begin{abstract}
It is now well documented that both increased and decreased stress responses can profoundly affect cognition and behavior. This mini review presents possible neural mechanisms subserving stress effects on memory and aggression, particularly focusing on glucocorticoid (GC) hyper- and hypofunction. First, uncontrollable stress impedes hippocampal memory and long-term potentiation (LTP). Because the hippocampus is important for the stability of long-term memory and because LTP has qualities desirable of an information storage mechanism, it has been hypothesized that stressinduced alterations in LTP contribute to memory impairments. Recent evidence suggests a neuralendocrine network comprising amygdala, prefrontal cortex (PFC), and glucocorticoids may be involved in regulating stress effects on hippocampal mnemonic functioning. Second, antisocial aggressiveness correlates with chronically decreased glucocorticoid production, and this condition leads in rats to behavioral-autonomic deficits reminiscent of the human disorder. Glucocorticoid deficiency-induced antisocial aggressiveness results from functional changes in the PFC, medial and central amygdala, and altered serotonin and substance P neurotransmissions. Accordingly, a neurobiological understanding of how stress and glucocorticoid deficiency alter brain, cognition, and behavior is an important challenge facing modern neuroscience with broad implications for individual and social well-being.
\end{abstract}

\section{Keywords}

learning; memory; aggression; corticosterone; hippocampus; amygdala; prefrontal cortex; synaptic plasticity

\section{INTRODUCTION}

All organisms experience "demands" in their environments, such as predation (threats, danger), dominance (status, relationship), and competition for resources (food, shelter, mate). Through the process of natural selection, evolution has endowed organisms with stress response, which is a crucial component of the animal's defense mechanism.

Stress, as a modern scientific concept profoundly influenced by nearly half century of work by Hans Selye, ${ }^{1}$ describes any significant distressing situations (real or perceived) that require necessary psychophysiological readjustment or adaptation for well-being of the individual. ${ }^{2-}$ ${ }^{5}$ In recent decades, there has been an enormous interest in the manifold effects of stress on

Address for correspondence: Jeansok J. Kim, Ph.D., Department of Psychology and Program in Neurobiology \& Behavior, University of Washington, Seattle, WA 98020, USA. Voice: +1-206-616-2685; fax: +1-206-685-3157. jeansokk@u.washington.edu. 
brain and behavior, as uncontrollable (chronic and intense) stress has been implicated in myriad psychopathologies, including anxiety, depression, posttraumatic stress disorders (PTSDs), schizophrenia, and drug use relapse.

The pioneering work of Selye directed attention to the effects of glucocorticoid (GC) hyperfunction as a pathogenic factor. Recent evidence suggests, however, that the consequences of chronic glucocorticoid hypofunction are not less devastating, and this endocrine condition also leads to a series of psychopathologies including antisocial aggressiveness.

A common thread binding stress-associated disorders appears to be the glucocorticoidassociated alterations in gene expression. Because glucocorticoid effects on cognition and behavior are reliably observed in a diverse array of animals (ranging from fish to humans), animal models (such as rodents) provide valuable means to study common neurobiological substrates underlying behavioral changes linked to alterations in glucocorticoid production. ${ }^{2-}$ ${ }^{5}$ This admittedly selective mini-review serves to highlight particular viewpoints and findings (from the authors) concerning stress-induced alterations in the hippocampal memory system and glucocorticoid deficiency-related changes in aggressive behavior. The highlighted findings may provide insights into understanding various stress-related disorders that severely limit the quality of human life in today's increasingly hectic and long-living society.

\section{Stress Effects on Memory and Plasticity}

Stress and Hippocampal Memory-The hippocampus is crucial for the stable formation of declarative/explicit memory in humans and spatial/relational memory in rodents. ${ }^{6-8}$ It is also involved in inhibiting stress-related hypothalamus-pituitary-adrenal (HPA) axis activity. ${ }^{2-5}$ As a high concentration of receptors for corticosteroids - the principal glucocorticoid synthesized by the adrenal cortex and secreted in abundance in response to stress (cortisol in human; corticosterone in rodent; CORT) - is localized in the hippocampus, the structure is quite susceptible to stress. This susceptibility has implications for its non-stress-related mnemonic functioning. Supporting this view are findings that stress generally impairs hippocampal memory tasks. In humans, impairments in verbal recall tasks have been observed in (i) PTSD patients ${ }^{9}, 10$; (ii) people with hypercortisolemia conditions, such as Cushing's disease and chronic depression ${ }^{11}$; and (iii) subjects administered high doses of CORT or stress. 12 In rodents, stress generally induces deficits in spatial memory tasks. ${ }^{4,13}$

Stress Effects on Hippocampal Plasticity-A number of stress-associated changes that can potentially influence mnemonic functioning have been identified in the hippocampus, such as (transient) alterations in the motivation-arousal-emotion systems, (relatively long-lasting) modifications in long-term potentiation (LTP), morphological changes, neuronal endangerment, and suppression of adult neurogenesis. ${ }^{3}$ Among these, the stress-induced impairment in LTP has garnered a particular interest as LTP is the leading candidate cellular model of information storage in the mammalian brain. ${ }^{14}$ Paralleling the behavioral data, in vitro and in vivo studies indicate that stress impairs LTP. ${ }^{15-20}$ Originally, hippocampal slices from rats that experienced 30-min restraint +30 intermittent tailshocks were shown to exhibit LTP deficits in the Schaffer collateral/commissural-CA1 pathway. ${ }^{15}$ Subsequent studies established that the LTP impairment is mainly due to psychological, and not physical (e.g., pain), aspects associated with stress. ${ }^{16,19}$ The LTP deficit is also observed in the dentate gyrus, and persists up to $48 \mathrm{~h}$ in rats (following an acute stress). There also seems to be a critical stress dosage requirement as 10 shocks (which robustly produce fear conditioning and elevate CORT) do not impair LTP. ${ }^{16}$ Recent studies further indicate that stress produces a time-dependent biphasic effect on LTP (an immediate enhancing effect followed by a longer-lasting inhibitory 
effect on LTP), ${ }^{21}$ and the same stress that impairs LTP enhances long-term depression (LTD; an additional synaptic model of memory) in the hippocampus. ${ }^{17}$

The discovery that stress impairs LTP is significant in two ways. ${ }^{22}$ First, it offers a testable substrate to investigate the phenomenon of stress-induced memory deficits; that is, if the notion that changes in synaptic efficacy is essential for memory is correct [Hebb's postulate ${ }^{23}$ ], then LTP impairments associated with stress might explain stress-induced memory deficits (Fig. 1A). Second, the LTP impairment can serve as a standard neural marker to compare behavioral effects resulting from the use of diverse "putative" stressors across laboratories. Not all stress paradigms would be expected to alter LTP and behavior in similar manners. Thus, the problem of qualifying, quantifying, or scaling different stressors and their behavioral effects can be normalized by examining LTP. ${ }^{22}$

Glucocorticoid Effects on Hippocampus-In most stress-hippocampus research, the usual strategy has been to relate the glucocorticoid level directly to stress effects, an approach that unveiled enormously useful information. ${ }^{2-5}$ Seminal rodent work has shown that the hippocampus is enriched with both mineralocorticoid receptors (MRs) and glucocorticoid receptors (GRs), ${ }^{24}$ and that CORT actions through these receptors mediate several stress effects on the hippocampus. Importantly, a dual relationship between the level of CORT and the magnitude of LTP has been described, where both low (via adrenalectomy, ADX) and high (via administration) levels of CORT are associated with impaired LTP. ${ }^{25}$ Other studies showed that selective activation of MRs increases LTP while added activation of GRs attenuates LTP. ${ }^{26}$ This suggests that basal (low) levels of CORT enhance potentiation through preferential stimulation of the high-affinity MRs and, during stress GR stimulation becomes important because levels of CORT become high enough to saturate low-affinity GRs. ${ }^{2}$ Behavioral studies found similar results—-spatial memory is impaired with GR, but not MR, activation. ${ }^{27}$

If CORT is the main contributing factor from behavioral stress, then removing it (during stress) and directly applying it (sans stress) should preclude and produce stress effects, respectively. However, there are data inconsistent with this simple linear CORT-stress effect notion. For instance, LTP is further reduced in ADX rats following stress and is not restored by CORT replacement. ${ }^{28}$ In intact animals administered dexamethasone (a synthetic GC that binds to GRs and by mimicking feedback suppresses the pituitary-adrenal response to stress), stressinduced LTP impairments occurred nonetheless, suggesting that the elevated GC level is not the whole mechanism whereby stress suppresses LTP. ${ }^{29}$ Notably, rats that experienced but were able to terminate shocks (i.e., control over shock cessation) did not show LTP impairments unlike "yoked" animals receiving identical shocks without control, although CORT levels were elevated equally in both groups. ${ }^{16}$ Recently, male rats with stress equivalent levels of CORT — via administration of CORT or exposure to a sexually receptive female-were found not impaired in spatial memory. ${ }^{30}$ Thus, CORT is unlikely an invariant physiological measure of stress since other sources (such as sex, exercise) can also significantly elevate it. Collectively, these data indicate that the elevated level of CORT is not a sufficient condition to reproduce stress effects on the hippocampus. Instead, it is likely that other factors (besides GC) constitute the central stress network that exerts cognitive influences. ${ }^{3}$

Amygdala and Prefrontal Cortex (PFC) Involvement in Stress-Evidence from anatomical, physiological, and behavioral studies indicates that the amygdala sends projections to the hippocampus, is involved in various stress-related behaviors (such as gastric erosion, analgesia, anxiety), alters the magnitude of perforant path-dentate gyrus LTP, and modulates the strength of hippocampal memory formation. ${ }^{31,32}$ Consistent with these results, recent studies found that amygdalar lesions and inactivation effectively block stress-induced impairments in hippocampal LTP and spatial memory. ${ }^{18}$ 
It is also plausible that the massive reciprocal connection between frontal cortical regions to "core" limbic structures regulates stress-hippocampal interactions. Of particular interest are the projections from the medial PFC (prelimbic and dorsal anterior cingulate cortices) to the amygdala and hippocampus. Specifically, the mPFC sends projections to the amygdala. ${ }^{33,34}$ The mPFC also sends projections to the hippocampal formation, via the entorhinal area and thalamus (nucleus reunions). ${ }^{35,36}$ The functional implication of these connections is that during stress "higher level" processing occurring in the MPFC can influence the hippocampus through multiple routes. Consistent with this view are findings that $\mathrm{mPFC}$ activity closely correlates with controllability, that is, inhibition (or extinction) of aversively motivated behavior. ${ }^{37,38} \mathrm{In}$ humans, damage to this structure (or hyporesponsive mPFC) results in impulsiveness (loss of controllability) and inappropriate emotional behavior in the absence of intellectual deficits. ${ }^{39}$

We recently tested the notion that $\mathrm{mPFC}$ exerts inhibitory influences on stress. Rats with mPFC lesions received either 20-min restraint +20 shocks (ineffective stress), 60-min restraint +60 shocks (effective stress), or 90-min restraint +90 shocks (>effective stress) (Fig. 1C). In intact animals, CA1 LTP was significantly observed in slices from control (No stress) and ineffective 20-min stress groups but not from effective 60-min and 90-min stress groups. In PFC-lesioned animals, the ineffective 20-min stress now reliably impaired LTP. Behaviorally, in rats with $\mathrm{mPFC}$ lesions, the same ineffective stress procedure also impaired long-term spatial memory. These findings suggest that mPFC normally exerts inhibitory stress influence (consistent with the view that a key function of mPFC is "controllability"), and that damage to this structure exacerbates stress effects on hippocampal LTP.

Need for a Systems Level Analysis of Stress-To fully understand the detrimental neurocognitive consequences of stress, it is vital to identify the basic elements (or controlling antecedent conditions) of stress and their neurobiological substrates. ${ }^{22}$ Recently, Kim and Diamond ${ }^{4}$ proposed that stress must satisfy three criteria: (i) it should generate heightened excitability/arousal (a variable denoted " $E$ "); (ii) it should induce perceived aversiveness (a variable " $A$ "); and (iii) it should produce uncontrollability (a variable " $U$," an inverse of controllability). At the simplest conceptual level stress $(S)$ can be represented: $S=E \times A \times$ $U$. The magnitude of neurocognitive stress effects is then determined by the dynamic (and not necessarily orthogonal) interactions between $E, A$, and $U$, which can be adjusted by varying the level of each variable. Figure $2 B$ illustrates a connectionist version with CORT, $A M Y G$ (amygdala), and $m P F C$ representing neurobiological counterparts of excitability $(E)$, aversiveness $(A)$, and controllability $(C)$, respectively. As mentioned earlier, these biological designations are supported by the evidence that: (i) CORT levels correlate with excitability/ arousal; (ii) amygdalar lesion/inactivation reduces aversive behavior while stimulation evokes aversive behavior; (iii) the mPFC activity correlates with controllability while damaged (or hyporesponsive) mPFC results in impulsiveness or loss of controllability; and (iv) CORT, AMYG, and $\mathrm{mPFC}$ are all responsive to stress.

In summary, identifying and rigorously testing the rules and design features of the systems level model of stress could provide an important foundation for developing a more comprehensive (qualitative and quantitative) understanding of the way in which stress influences neurocognition and contributes to a variety of stress-related disorders.

\section{Glucocorticoid Deficiency and Aggression}

Glucocorticoid Status and Antisocial Aggressiveness-It is now widely accepted that not only increased but also decreased GC signaling leads to pathological conditions. ${ }^{40}$ To date, reduced plasma GCs were associated with antisocial personality disorder and its childhood antecedent conduct disorder, PTSD, fibromyalgia, chronic fatigue, and burnout. ${ }^{41-44}$ In contrast to PTSD, where the effects of GC deficiency appear to be mediated by a 
secondary upregulation of noradrenergic and CRH signaling, ${ }^{45}$ antisocial behavior develops in conjunction with low autonomic responsiveness. ${ }^{46}$ Thus, decreased plasma GCs may underlay a series of psychopathologies, and the nature of the disorder may also depend on associated neural changes (e.g., increased or decreased noradrenergic neurotransmission).

In an attempt to develop a model of antisocial aggressiveness, we studied adrenalectomized rats, in which low and stable levels of GRs were ensured by subcutaneous corticosterone pellets (ADXr). Mimicking in rats the endocrine condition associated with antisocial aggressiveness in humans (i.e., low GC levels) resulted in the development of three important symptoms of the disorder: (i) antisocial type of aggressiveness (attacks aimed at vulnerable body parts of opponents), (ii) low autonomic arousal during fights, and (iii) social deficits. ${ }^{47,48}$

Laboratory models are usually evaluated by investigating their face, construct, and predictive validity. ${ }^{49}$ Considering the phenomenological similarities between human antisocial aggressiveness and the behavior of our rats, one can assume that the face validity of the model is acceptable. Construct validity is suggested by the shared GC background. Predictive validity is more problematic, as no reliably efficient treatments are known for antisocial personality disorder. Nevertheless, earlier findings suggested that serotonergic agents act in a rather similar fashion in antisocial personality-disordered people and rats submitted to our model..$^{50-53}$ This similarity indicates that the model has a certain degree of predictive validity.

The Neural Background of Antisocial Aggressiveness in Rats-By comparing the neural background of glucocorticoid deficiency-induced violent and normal (territory-related) aggression, we identified four areas that may be responsible for the development of abnormal attacks: the PFC, medial amygdala (MeA), central amygdala (CeA), and the raphe.

In the first series of experiments, we investigated aggression-induced neuronal activation by labeling the c-Fos protein. Aggression per se activated all the brain areas that were earlier believed to control aggression. As compared to controls, glucocorticoid deficiency-induced violent aggression induced a dramatic increase in the activation of the CeA that is tightly bound to the control of fear. ${ }^{54}$ To our surprise, no significant differences were seen in other aggression-related brain areas. We assumed, however, that the changes seen in c-Fos studies were accompanied by more subtle alterations that cannot be revealed by a simple c-Fos staining. Therefore, the next series of experiments aimed at the neurochemical identification of neurons that were activated by the execution of attacks. This approach identified three other brain areas that may be responsible for the execution of violent attacks: the raphe, PFC, and MeA.

In rats submitted to resident/intruder conflicts (normal-territorial-aggression), the activation of serotonergic neurons showed a significant negative correlation with attack counts. In line with earlier findings, this suggested that the activation of serotonergic neurons downregulates attacks. This correlation, however, was lost in rats submitted to the violent aggression model, suggesting that the serotonergic control of aggression is lost in glucocorticoid-deficient rats $^{52}$ (Fig. 2, upper panel). This finding was confirmed later by pharmacological experiments, where the effect of the serotonergic anxiolytic buspirone was dramatically altered in GCdeficient, as compared to sham-operated, rats. ${ }^{53}$

In the PFC, we double labeled neurons for activation (e.g., c-Fos) and neurochemical markers. ${ }^{55}$ Glucocorticoid deficiency markedly and specifically decreased the activation of CCKcontaining GABAergic interneurons that control the inputs of glutamatergic principal cells, and ensure the fine-tuning of their function. ${ }^{56}$ A multiple regression analysis showed that normal and violent attacks were associated with different patterns of principal cell activation when all the relevant PFC subareas were analyzed together (Fig. 2, middle panel). Moreover, 
the specific pattern seen in GC-deficient rats predicted the expression of vulnerable attack by a precision that exceeded $90 \%$.

Finally, we investigated the activation of NK1 receptor-expressing neurons in brain areas relevant to aggression control. The reason for performing these studies was that substance $\mathrm{P}$ and its NK1 receptor was implicated in the defensive rage of cats. ${ }^{57}$ The activation of NK1 receptor-expressing neurons was significantly stronger in GC-deficient rats and correlated significantly with the execution of violent attacks ${ }^{58}$ (Fig. 2, lower panel). Moreover, NK1 receptor blockade specifically decreased violent aggression, without affecting more normal forms of aggressiveness. ${ }^{58}$

\section{CONCLUSIONS}

Psychopathologies may not only be associated with GC excess, but also with GC deficiency. In antisocial aggressiveness, GC hypofunction is associated with reduced autonomic activation and social deficits. Our laboratory findings suggest that GC deficiency and human antisocial aggressiveness are causally linked, as mimicking the former in rats resulted in behavioral symptoms strongly reminiscent of the latter. It occurs that chronic GC deficiency leads to neural changes that are tightly bound to the execution of antisocial attacks in rats. Such changes were identified in restricted brain regions, namely the raphe, PFC, as well as the central and medial amygdala. Noteworthy, these brain areas are strongly interrelated: serotonergic neurons have a strong effect on prefrontal functions, which tightly control the amygdala. We suggest that studying the impact of GC deficiency on behavior and brain function reveals important aspects of the mechanisms that underlay GC deficiency-associated behavioral disorders; moreover, such studies may identify novel treatment strategies.

\section{References}

1. Selye H. A syndrome produced by diverse nocuous agents. Nature 1936;138:32.

2. McEwen BS, Sapolsky RM. Stress and cognitive function. Curr Opin Neurobiol 1995;5:205-216. [PubMed: 7620309]

3. Kim JJ, Yoon KS. Stress: metaplastic effects in the hippocampus. Trends Neurosci 1998;21:505-509. [PubMed: 9881846]

4. Kim JJ, Diamond DM. The stressed hippocampus, synaptic plasticity and lost memories. Nat Rev Neurosci 2002;3:453-462. [PubMed: 12042880]

5. Diamond DM, Cambell AM, Park CR, et al. The temporal dynamics model of emotional memory processing: a synthesis on the neurobiological basis of stress-induced amnesia, flashbulb and traumatic memories, and the Yerkes-Dodson law. Neural Plasticity 2007;2007:1-33.

6. Scoville WB, Milner B. Loss of recent memory after bilateral hippocampal lesions. J Neurol Neurosurg Psychiatry 1957;20:11-21. [PubMed: 13406589]

7. O'Keefe, J.; Nadel, L. The Hippocampus as a Cognitive Map. Oxford University Press; New York NY: 1978.

8. Eichenbaum H. A cortical-hippocampal system for declarative memory. Nat Rev 2000;1:41-50.

9. Bremner JD, Scott TM, Delaney RC, et al. Deficits in short-term memory in posttraumatic-stressdisorder. Am J Psychiatry 1993;150:1015-1019. [PubMed: 8317569]

10. Utto M, Vasterling JJ, Brailey K, Sutker PB. Memory and attention in combat-related posttraumaticstress-disorder (PTSD). J Psychopathol Behav Assess 1993;15:43-52.

11. Starkman MN, Gebarski SS, Berent S, Schteingart DE. Hippocampal formation volume, memory dysfunction, and cortisol levels in patients with Cushing's syndrome. Biol Psychiatry 1992;32:756765. [PubMed: 1450290]

12. Newcomer JW, Craft S, Hershey T, et al. Glucocorticoid-induced impairment in declarative memory performance in adult humans. J Neurosci 1994;14:2047-2053. [PubMed: 8198631] 
13. Diamond DM, Park CR, Heman KL, Rose GM. Exposing rats to a predator impairs spatial working memory in the radial arm water maze. Hippocampus 1999;9:542-52. [PubMed: 10560925]

14. Bliss TVP, Collingridge GL. A synaptic model of memory: long-term potentiation in the hippocampus. Nature 1993;361:31-39. [PubMed: 8421494]

15. Foy MR, Stanton ME, Levine $S$, Thompson RF. Behavioral stress impairs long-term potentiation in rodent hippocampus. Behav Neural Biol 1987;48:138-149. [PubMed: 2820370]

16. Shors TJ, Seib TB, Levine S, Thompson RF. Inescapable versus escapable shock modulates longterm potentiation in the rat hippocampus. Science 1989;244:224-226. [PubMed: 2704997]

17. Kim JJ, Foy MR, Thompson RF. Behavioral stress modifies hippocampal plasticity through $N$-methylD-aspartate receptor activation. Proc Natl Acad Sci 1996;93:4750-4753. [PubMed: 8643474]

18. Kim JJ, Koo JW, Lee HJ, Han JS. Amygdalar inactivation blocks stress-induced impairments in hippocampal long-term potentiation and spatial memory. J Neurosci 2005;25:1532-1539. [PubMed: 15703407]

19. Diamond DM, Fleshner M, Rose GM. Psychological stress repeatedly blocks hippocampal primed burst potentiation in behaving rats. Behav Br Res 1994;62:1-9.

20. Diamond DM, Park CR. Predator exposure produces retrograde amnesia and blocks synaptic plasticity. Progress toward understanding how the hippocampus is affected by stress. Ann N Y Acad Sci 2000;911:453-455. [PubMed: 10911891]

21. Akirav RLG. Biphasic modulation of hippocampal plasticity by behavioral stress and basolateral amygdalar stimulation in the rat. J Neurosci 1999;19:10530-10535. [PubMed: 10575049]

22. Kim JJ, Song EY, Kosten TA. Stress effects in the hippocampus: synaptic plasticity and memory. Stress: Internatl. J Biol Stress 2006;9:1-11.

23. Hebb, DO. The Organization of Behavior: A Neuropsychological Theory. Wiley; New York, NY: 1949.

24. Reul JMHM, de Kloet ER. Two receptor systems for corticosterone in rat brain: microdistribution and differential occupation. Endocrinology 1985;117:2505-2511. [PubMed: 2998738]

25. Diamond DM, Bennett MC, Fleshner M, Rose GM. Inverted-U relationship between the level of peripheral corticosterone and the magnitude of hippocampal primed burst potentiation. Hippocampus 1992;2:421-430. [PubMed: 1308198]

26. Pavlides C, Watanabe Y, Margarinos AM, Mcewen BS. Opposing roles of type I and type II adrenal steroid receptors in hippocampal long-term potentiation. Neuroscience 1995;68:387-394. [PubMed: 7477948]

27. Conrad CD, Lupien SJ, McEwen BS. Support for a bimodal role for type II adrenal steroid receptors in spatial memory. Neurobiol Learn Mem 1999;72:39-46. [PubMed: 10371714]

28. Shors TJ, Levine S, Thompson RF. Effect of adrenalectomy and demedullation on the stress-induced impairment of long-term potentiation. Neuroendocrinology 1990;51:70-75. [PubMed: 2106090]

29. Foy MR, Levine S, Thompson RF. Manipulation of pituitary-adrenal activity affects neural plasticity in rodent hippocampus. Psychol Sci 1990;3:201-204.

30. Woodson JC, Macintosh D, Fleshner M, Diamond DM. Emotion-induced amnesia in rats: working memory-specific impairment, corticosterone-memory correlation, and fear versus arousal effects on memory. Learn Mem 2003;10:326-336. [PubMed: 14557605]

31. LeDoux, JE. The Emotion Brain. Simon \& Schuster; New York, NY: 1996.

32. McGaugh JL. Memory-a century of consolidation. Science 2000;287:248-251. [PubMed: 10634773]

33. Heidbreder CA, Groenewegen HJ. The medial prefrontal cortex in the rat: evidence for a dorsalventral distinction based upon functional and anatomical characteristics. Neurosci Biobehav Rev 2003;27:555-579. [PubMed: 14599436]

34. McDonald AJ. Cortical pathways to the mammalian amygdala. Prog Neurobiol 1998;55:257-332. [PubMed: 9643556]

35. Hurley K, Herbert H, Moga MM, Saper CB. Efferent projections of the infralimbic cortex of the rat. J Comp Neurol 1991;308:249-276. [PubMed: 1716270]

36. Sesack SR, Deutch AY, Roth RH, Bunney BS. Topographical organization of the efferent projections of the medial prefrontal cortex in the rat: an anterograde tract-tracing study using Phaseolus vulgaris Leucoagglutinin. J Comp Neurol 1989;290:213-242. [PubMed: 2592611] 
37. Wellman CL, Izquierdo A, Garrett JE, et al. Impaired stress-coping and fear extinction and abnormal corticolimbic morphology in serotonin transporter knock-out mice. J Neurosci 2006;27:684-691. [PubMed: 17234600]

38. Maier SF, Amat J, Maratta MV, et al. Behavioral control, the medial prefrontal cortex, and resilience. Dialog Clin Neurosci 2006;8:397-406.

39. McNally RJ. Cognitive abnormalities in post-traumatic stress disorder. Trends Cog Sci 2006;10:271277.

40. Raison CL, Miller AH. When not enough is too much: the role of insufficient glucocorticoid signaling in the pathophysiology of stress-related disorders. Am J Psychiatry 2003;160:1554-1565. [PubMed: 12944327]

41. Crofford LJ, Pillemer SR, Kalogeras KT, et al. Hypothalamic-pituitary-adrenal axis perturbations in patients with fibromyalgia. Arthritis Rheum 1994;37:1583-1592. [PubMed: 7980669]

42. Demitrack MA, Dale JK, Straus SE, et al. Evidence for impaired activation of the hypothalamicpituitary-adrenal axis in patients with chronic fatigue syndrome. J Clin Endocrinol Metab 1991;73:1224-1234. [PubMed: 1659582]

43. Virkkunen M. Urinary free cortisol secretion in habitually violent offenders. Acta Psychiatr Scand 1985;72:40-44. [PubMed: 2994368]

44. Yehuda R. Biology of posttraumatic stress disorder. J Clin Psychiatry 2001;62(Suppl 17):41-46. [PubMed: 11495096]

45. Baker DG, West SA, Nicholson WE, et al. Serial CSF corticotropin-releasing hormone levels and adrenocortical activity in combat veterans with posttraumatic stress disorder. Am J Psychiatry 1999;156:585-588. [PubMed: 10200738]

46. Raine A. Autonomic nervous system factors underlying disinhibited, antisocial, and violent behavior. Biosocial perspectives and treatment implications. Ann N Y Acad Sci 1996;794:46-59. [PubMed: 8853591]

47. Haller J, Halasz J, Mikics E, Kruk MR. Chronic glucocorticoid deficiency-induced abnormal aggression, autonomic hypoarousal, and social deficit in rats. J Neuroendocrinol 2005;16:550-557. [PubMed: 15189330]

48. Haller J, Kruk MR. Normal and abnormal aggression: human disorders and novel laboratory models. Neurosci Biobehav Rev 2006;30:292-303. [PubMed: 16483889]

49. Willner P. Validation criteria for animal models of human mental disorders: learned helplessness as a paradigm case. Prog Neuropsychopharmacol Biol Psychiatry 1986;10:677-690. [PubMed: 3809518]

50. Kutcher SP, Marton P, Korenblum M. Relationship between psychiatric illness and conduct disorder in adolescents. Can J Psychiatry 1989;34:526-529. [PubMed: 2766205]

51. Whitehead PD. Causality and collateral estoppel: process and content of recent SSRI litigation. J Am Acad Psychiatry Law 2003;31:377-382. [PubMed: 14584540]

52. Haller J, Toth M, Halasz J. The activation of raphe serotonergic neurons in normal and hypoarousaldriven aggression: a double labeling study in rats. Behav Brain Res 2005;161:88-94. [PubMed: 15904714]

53. Haller J, Horvath Z, Bakos N. The effect of buspirone on normal and hypoarousal-driven abnormal aggression in rats. Prog Neuropsychopharmacol Biol Psychiatry 2007;31:27-31. [PubMed: 16893596]

54. Halasz J, Liposits Zs, Kruk MR, Haller J. Neural background of glucocorticoid dysfunction-induced abnormal aggression in rats: involvement of fear- and stress-related structures. Eur J Neurosci 2002;15:561-569. [PubMed: 11876784]

55. Halasz J, Toth M, Kallo I, et al. The activation of prefrontal cortical neurons in aggression-A double labeling study. Behav Brain Res 2006;175:166-175. [PubMed: 16978716]

56. Freund TF. Interneuron diversity series: Rhythm and mood in perisomatic inhibition. Trends Neurosci 2003;26:489-495. [PubMed: 12948660]

57. Shaikh MB, Steinberg A, Siegel A. Evidence that substance P is utilized in medial amygdaloid facilitation of defensive rage behavior in the cat. Brain Res 1993;625:283-294. [PubMed: 7506110]

58. Halasz J, Toth M, Mikics E, et al. The effect of NK1 receptor blockade on territorial aggression and in a model of violent aggression. Biol Psych. 2007In press 
(A)

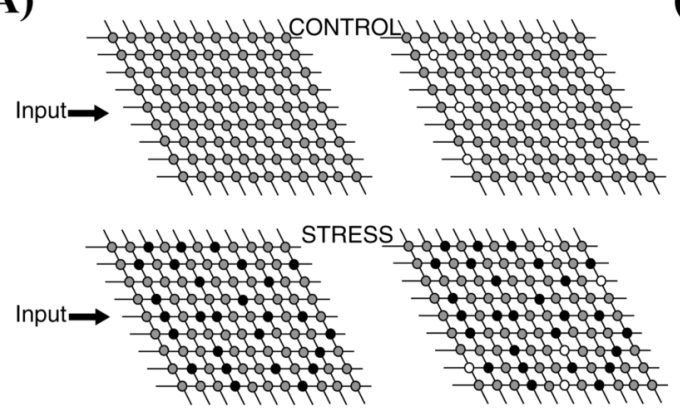

(C)

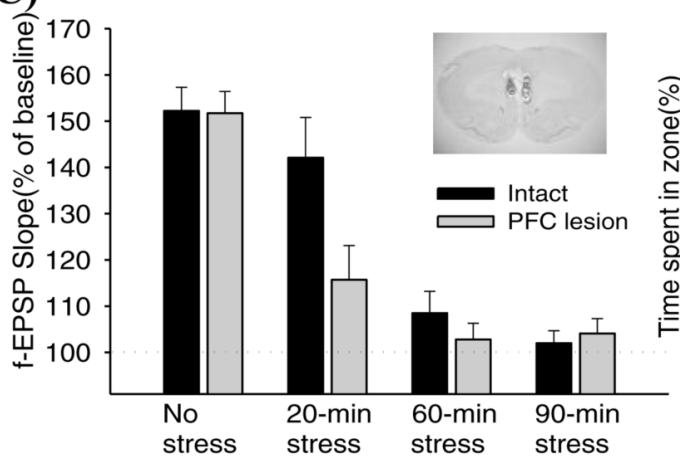

(B)

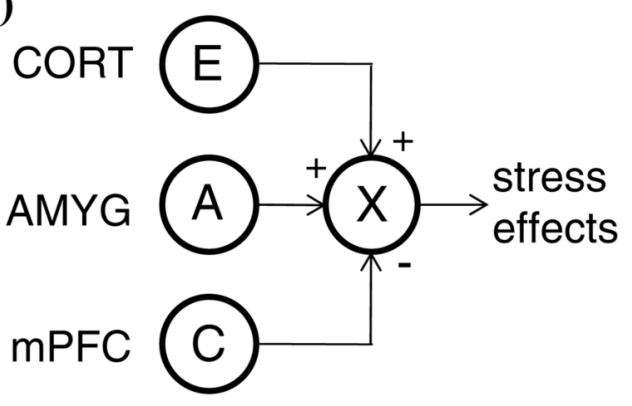

FIGURE 1.

(A) An illustration of how stress-induced impairments in LTP can impede subsequent memory in the hippocampus. The gray circles on the matrices represent synapses with normal capacity to undergo plasticity (e.g., LTP), thereby supporting normal memory configuration (white circles). The black circles represent synapses with altered properties of plasticity (e.g., impaired LTP), which impair subsequent memory processing. (B) A simple connectionist model showing CORT (excitability, E), AMYG (aversiveness, A), and mPFC (controllability, C) interaction to produce stress effects. The model posits that CORT and AMYG exert excitatory $(+)$ and $\mathrm{mPFC}$ exerts inhibitory (-) stress influences. $\mathrm{X}$ denotes a target structure, such as the hippocampus. (C) Electrolytic mPFC lesions and stress effects on LTP and spatial memory. In no stress condition, LTP (measured 40-60-min posttetanus) was robustly observed in hippocampal slices prepared from intact and mPFC-lesioned rats. Both 60-min and 90-min stress reliably impaired LTP in intact and mPFC-lesioned rats. While 20-min stress was ineffective in intact rats, it effectively reduced LTP in mPFC-lesioned animals. The inset shows an example of mPFC-lesioned brain section. Percentage of time spent swimming in the platform (represented by a dotted circle) quadrant zone during the 60 -sec probe test. Sham-No stress, Sham-20-min (ineffective) stress, and mPFC-No stress groups exhibited reliable spatial memory, whereas Sham-60-min (effective) stress, mPFC-20-min stress, and mPFC-60-min stress groups did not. 

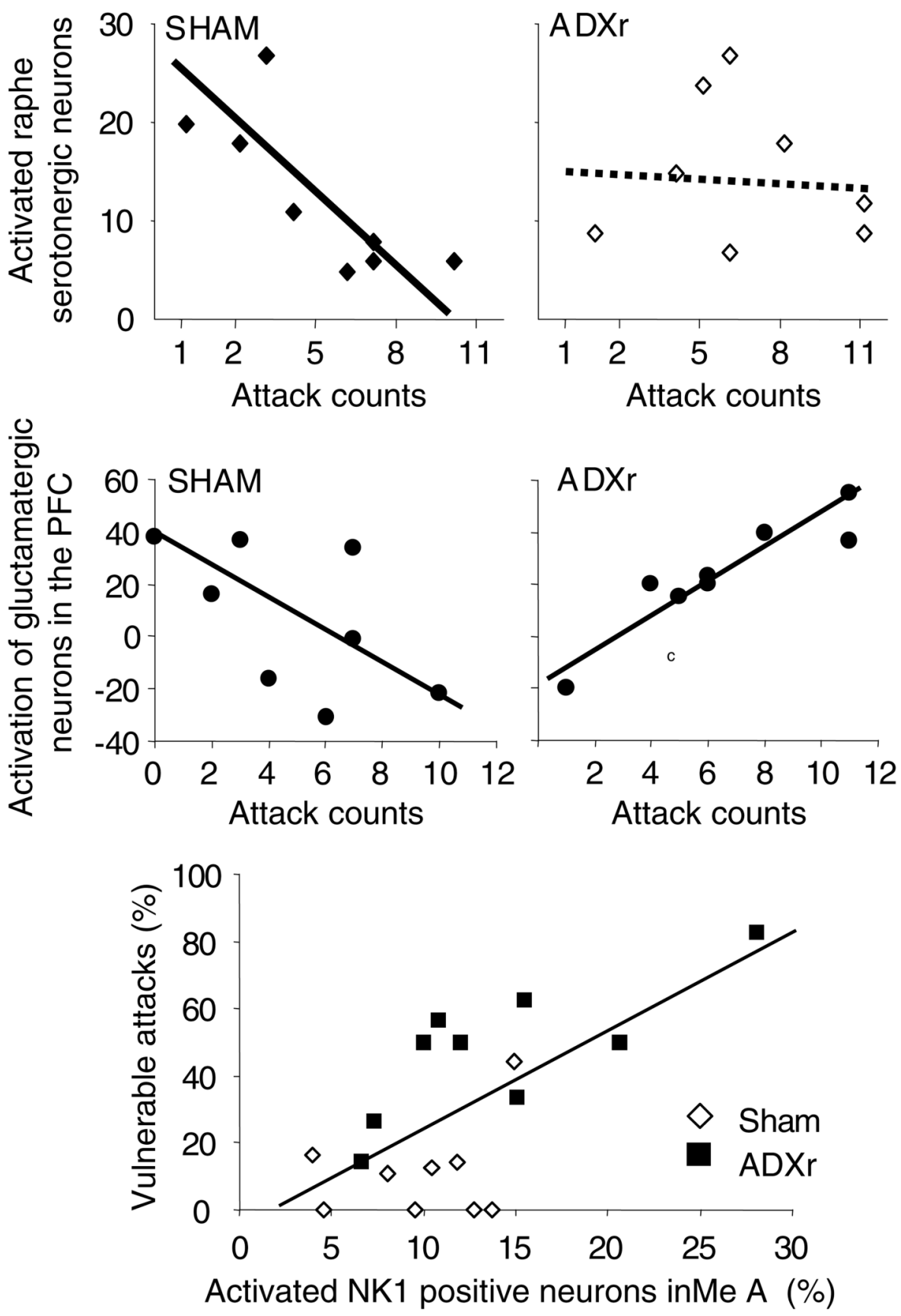

FIGURE 2.

Correlations between neuronal activation and the execution of attacks. In controls (Sham), serotonergic- and marginally, prefrontal glutamatergic activation correlated negatively with the execution of attacks, whereas no significant correlation was seen between vulnerable attacks and NK1 receptor-expressing neurons of the medial amygdala (MeA). This picture changed dramatically in glucocorticoid-deficient ("violent", ADXr) rats: serotonergic neurons appeared to lose their role in controlling attacks, whereas PFC glutamatergic and MeA NK1 cell activation correlated positively with the execution of attacks and vulnerable attacks, respectively. 